\title{
Une nouvelle espèce de Turraea (Meliaceae) du Sud-Ouest de Madagascar
}

\author{
Tabita N. Randrianarivony, Monique F. Randriatsivery, \\ Tefy H. Andriamihajarivo \& Martin W. Callmander
}

\begin{abstract}
RANDRIANARIVONY, T.N., M.F. RANDRIATSIVERY,T.H. ANDRIAMIHAJARIVO \& M.W. CALLMANDER (2017). A new species of Turraea (Meliaceae) from Southwestern Madagascar. Candollea 72: 283-288. In French, English and French abstracts. DOI: http://dx.doi. org/10.15553/c2017v722a5

A new species of Turraea L. (Meliaceae) is described from Southwestern Madagascar: Turraea cauliflora J.-F. Leroy \& Lescot ex Randrianari. \& Callm. The new species is endemic to the Analavelona massif, which is a sacred forest to local population and recently became a protected area. The Analavelona region exhibits a unique subhumid vegetation in an area dominated by dry habitats. The new species differs from the other Malagasy species of Turraea by its cauliflorous inflorescence and its pubescence on the peduncle and fruit. Line drawings are provided, along with a discussion of its morphological affinities and a preliminary assessment of its risk of extinction based on IUCN Red List Categories and Criteria.
\end{abstract}

\section{Résumé}

RANDRIANARIVONY, T.N., M.F. RANDRIATSIVERY,T.H. ANDRIAMIHAJARIVO \& M.W. CALLMANDER (2017). Une nouvelle espèce de Turraea (Meliaceae) du Sud-Ouest de Madagascar. Candollea 72: 283-288. En français, résumés anglais et français. DOI: http://dx.doi. org/10.15553/c2017v722a5

Une nouvelle espèce de Turraea L. (Meliaceae) est décrite du Sud-Ouest de Madagascar: Turraea cauliflora J.-F. Leroy \& Lescot ex Randrianari. \& Callm. La nouvelle espèce est endémique du massif forestier d'Analavelona qui est une forêt sacrée pour la population locale et est devenue récemment une aire protégée. La forêt d'Analavelona est une forêt subhumide dans une zone sèche du Sud-Ouest de Madagascar. Cette nouvelle espèce diffère des autres espèces du genre par ses inflorescences cauliflores, ainsi que par la pubescence de ses pédoncules et de ses fruits. Une illustration au trait est fournie, ainsi qu'une discussion de ses affinités morphologiques et une évaluation préliminaire de son risque d'extinction selon les Catégories et les Critères de l'UICN.

\section{Keywords}

MELIACEAE - Turraea - New species - Taxonomy - Analavelona sacred forest - Madagascar

\footnotetext{
Addresses of the authors:

TNR, THA: Missouri Botanical Garden, Research and Conservation Program, B.P. 3391, Antananarivo 101, Madagascar.

E-mail: tabita.randriannarivony@mobot-mg.org

MFR: Tany Meva, B.P. 4300, Antananarivo 103, Madagascar.

MWC: Conservatoire et Jardin botaniques de la Ville de Genève, C.P. 60, 1292 Chambésy, Suisse.

Submitted on December 23, 2016. Accepted on May 5, 2017.
}

First published online on June 24, 2017 


\section{Introduction}

Le genre Turraea L. (Meliaceae) est un genre paléotropical qui compte actuellement c. 65 espèces réparties principalement en Afrique (35 spp.; APD, 2017), aux Mascareignes (9 spp.; Scott \& Bosser, 1997; Bosser, 2002), à Madagascar et aux Comores (19 spp.; Madagascar Catalogue, 2017) et une seule espèce distribuée en Asie et en Australie (T. pubescens Hell.) (MAв вERLey et al., 1995; APC, 2017). Le genre Turraea est reconnaissable dans la famille des Meliaceae par ses feuilles simples spiralées, ses fleurs à étamines totalement soudées, un réceptacle pollinique (apex du style modifié) et son fruit à capsule déhiscente possédant une graine portant un arille (Pennington \& Styles, 1975).

Dix-neuf espèces sont actuellement acceptées à Madagascar (17 endémiques et 2 partagées avec les Iles des Comores) (Madagascar Catalogue, 2017). Cependant un manuscrit non publié de la Flore de Madagascar et des Comores commencé par Jean-François Leroy (1915-1999) puis poursuivi par Michèle Lescot (1939-) dénombre 33 espèces à Madagascar dont une grande partie reste encore inédite. Deux nouvelles espèces découvertes dans les montagnes du Nord de Madagascar ont été décrites plus récemment (CALlmander et al., 2012).

La forêt d'Analavelona se trouve dans le Sud-Ouest de Madagascar. Elle fait partie des aires protégées nouvellement créés à Madagascar. Le décret de cette forêt a été proclamé en avril 2015 comme "Aire Protégée de catégorie III (Monument naturel)» selon l'UICN (MEEF, 2015). Cette forêt est considérée comme «sacrée» par les villageois car ils croient que les âmes de leurs ancêtres y reposent (HorNing, 2004). La forêt d'Analavelona, oasis de forêt pluviale dans un milieu chaud et sec, culmine à $1350 \mathrm{~m}$ et couvre une surface de 4044 ha (ANDriamihajarivo, 2014). Le micro-climat subhumide (Moat \& Smith, 2007) permet le développement de quatre types de formations végétales: la formation sèche, la formation de transition, la formation sub-humide et le bush xérophile (RANDrianarivony, 2015). Cette mosaïque de végétations a permis d'inventorier plus de 400 espèces de plantes vasculaires dont $73 \%$ sont endémiques de Madagascar et quelques-unes sont endémiques locales (ANDriamihajarivo et al., 2008): Aloe analavelonensis Letsara et al. (Asphodelaceae), Oeceoclades analavelensis (H. Perrier) Garay \& P. Taylor (Orchidaceae), Dombeya ambalabeensis subsp. analavelonae (Arènes) Appleq. (Malvaceae).

Afin de compléter les données scientifiques nécessaires à une gestion durable et efficace de la forêt d'Analavelona, des études sur l'utilisation de cette forêt comprenant des inventaires botaniques ont été effectuées (RANDRIANARIVONY, 2015 ; RANDRIANARIVONy et al., 2016). Lors de ces inventaires, TNR en 2012, puis TNR et THA en 20152016, ont retrouvé une espèce cauliflore de Turraea dont les premières collections remontent à Henri Humbert en 1946 et
René Capuron en 1962. Ces nouvelles récoltes ont permis d'obtenir du matériel nouveau nous permettant aujourd'hui de décrire cette nouvelle espèce. Elle fait partie des nombreuses espèces restées inédites à la suite du travail de Leroy et Lescot sur les Meliaceae de Madagascar. Nous décrivons donc ici cette espèce emblématique de la forêt sacrée d'Analavelona(Fig. 1): Turraea cauliflora J.-F. Leroy \& Lescot ex Randrianari. \& Callm. Une illustration au trait est fournie ainsi qu'une carte de distribution, des photographies de terrain et une discussion sur ses affinités morphologiques ainsi que son statut préliminaire de conservation.

\section{Systématique}

Turraea cauliflora J.-F. Leroy \& Lescot ex Randrianari. \& Callm., spec. nova (Fig. 2, 3).

Typus: Madagascar. Prov. Toliara: bassin de la Menanadabe, massif de l'Analavelona, au N du Fiherenana, 1000-1300 m, 13-15.XII.1962, fr., Service Forestier 22188 (holo- : P [P00853048]!; iso-: G [G00341686]!, P [P00853049, P00853050]!, TEF).

Turrea cauliflora J.-F. Leroy $\mathcal{E}^{2}$ Lescot ex Randrianari. Eळ Callm. differs from the other members of the genus by its cauliflory, its pubescent leaves, its flowers with 5 sepals and petals, its staminal tube with no appendices, and the pubescent fruits with 12 valves.

Petit arbre cauliflore, de 4 à $7 \mathrm{~m}$ de haut. Rameaux à écorce grisâtre, rugueuse, à écailles très serrées, d'abord densément hirsute à longs poils dorés dressés devenant glabre. Bourgeons tomenteux à poils dorés. Limbe foliaire membraneux, $(5,9-)$ $6,5-9,4(-10) \times(3,2-) 3,6-6,3(-7) \mathrm{cm}$, à base cunéiforme à obtuse, courtement acuminé au sommet, elliptique, pubescent à longs poils blanc-brillants à dorés sur les deux faces et particulièrement sur les nervures primaires et secondaires, plus dense à la face inférieure; marge entière, parfois \pm dentelée dans la partie supérieure; nervure primaire en creux en dessus; nervures secondaires proéminentes en dessous, 8-10 paires; réticulum bien marqué sur les deux faces, angle de nervation $60^{\circ}$; pétiole court, 2-4 mm, tomenteux à poils dorés. Inflorescence multiflore. Boutons floraux obtus au sommet. Pédoncule grêle, long de 1,6-3,5 cm, hirsute à petits poils éparses, blancs, raides, dressés de 0,8 mm, \pm enchevêtrés. Fleurs longues de 2,5 cm. Calice cupuliforme, de 3,2 × $4 \mathrm{~mm}, 5$-dentés, les dents largement et courtement triangulaires-aigus, de 0,6 $\times 0,8 \mathrm{~mm}$, densément tomenteux à petits poils blancs raides, enchevêtrés à l'extérieur, glabre à l'intérieur. Corolle à préfloraison imbriquée, à 5 pétales membraneux, linéaires, obtus au sommet, sensiblement égal au tube staminal; pétale blanc de 1-1,6 cm, large de 1,5$2,2 \mathrm{~mm}$. Tube staminal entier, cylindrique, membraneux, long de 1,2-2 cm, glabre; appendices staminaux absents. 


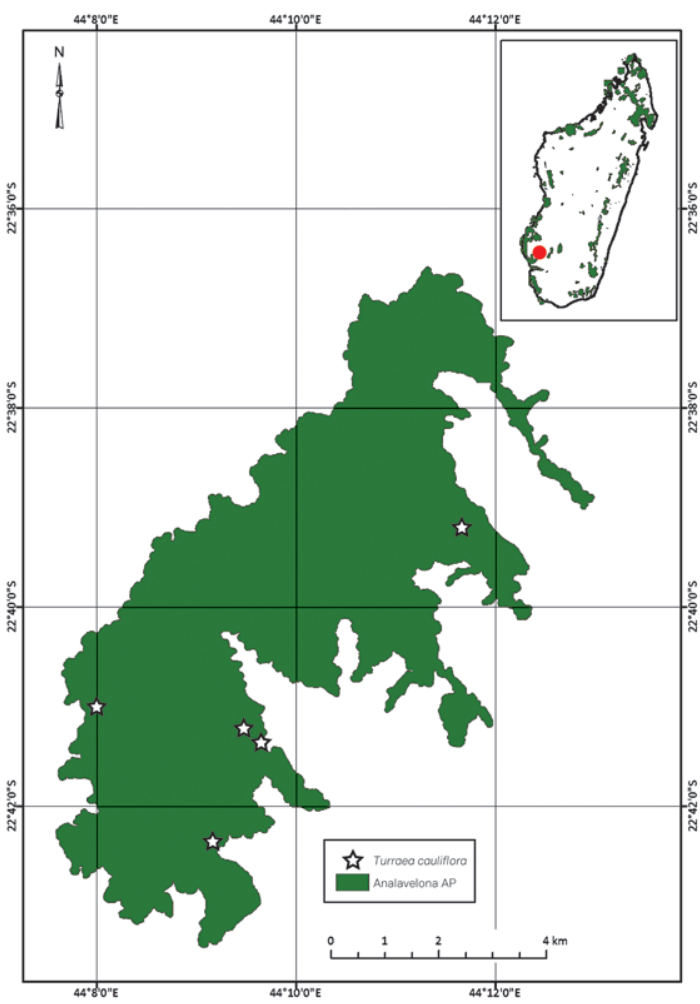

Fig. 1. - Carte de la forêt d'Analavelona montrant la distribution de Turraea cauliflora J.-F. Leroy \& Lescot ex Randrianari. \& Callm. (étoiles).

Anthères $10,2,3 \times 0,5 \mathrm{~mm}$, oblongues, à apicule aigu court $(0,2$ $0,3 \mathrm{~mm}$ ), glabres. Ovaire ellipsoïde, 1,2 ×0,8 $\mathrm{mm}$, hirsute à longs poils blanc-brillants (c. $1 \mathrm{~mm}$ ), à 10 loges à 2 ovules superposés, à placentation centrale. Style longuement exsert sur c. 1,9-3,2 cm. Réceptacle pollinique ellipsoïde, $4 \times 1 \mathrm{~mm}$; stigmate discoïde, $0,2 \times 2 \mathrm{~mm}$, à lobes stigmatiques réduits de $0,1 \times 0,5 \mathrm{~mm}$. Fruits caulinaires dès l'extrême base du tronc, globuleux, $0,9-1,5 \times 0,8 \mathrm{~cm}$, à $10-12$ valves souvrant sur toute leur longueur à maturité, à longs poils jaunes dorés, raides, très dense chez les jeunes fruits, moins dense chez les fruits matures; péricarpe de 0,2-0,5 $\mathrm{mm}$ d'épaisseur; calice persistant. Graines groupées vers le centre, réniformes, (5-)6 $\times(3-) 4 \mathrm{~mm}, 2$ graines soudées à la partie supérieure dans chaque loge; testa brunâtre, $0,1 \mathrm{~mm}$ d'épaisseur, plissé; arille fixé au niveau de la chalaze, jusqu'à $2 / 3$ à partir de l'extrémité supérieure au point de soudure des 2 graines, membraneux, mince.

Noms vernaculaires. - «Mampisaraky» (Andriamihajarivo et al. 1962, Randrianarivony et al. 463, 750); «Tobory» (Service forestier 22188).

Distribution, écologie et phénologie. - Turraea cauliflora est endémique de la forêt humide d'Analavelona. Elle a été récoltée dans la partie Est du massif forestier
d'Analavelona entre 900 et $1100 \mathrm{~m}$ d'altitude (Fig. 1) sur un substrat basaltique issu du volcanisme du Crétacé (Sourdat, 1976). Des boutons floraux ainsi que des fleurs séchées ont été collectés avec les échantillons en fruits en décembre. La floraison de cette espèce débute dès le mois de novembre et la fructification intervient entre décembre et février.

Statut de conservation. - Turraea cauliflora occupe une zone d'occurrence (EOO) estimée à c. $11 \mathrm{~km}^{2}$ dans quatres localités distinctes de la forêt d'Analavelona. Malgré la protection récente de cette forêt (voir ANDriaminajarivo, 2014), cette nouvelle espèce à distribution très restreinte est menacée par des feux annuels et par la coupe illicite. Turraea cauliflora est donc provisoirement considérée comme «Vulnérable» [VU D2] selon les Critères et les Catégories de la Liste Rouge de l'UICN (IUCN, 2012).

Notes. - Turraea cauliflora est la seule espèce de Turraea cauliflore possèdant des fleurs et fruits insérés à l'extrémité basale du tronc connue de Madagascar (Fig. 2, 3). Parmi les espèces pubescentes (T. fockei Buchenau, T. humbertii Danguy, T. sericea Sm, T. rostrata C. DC, T. thouvenotii Danguy et $T$. virens L.), la nouvelle espèce s'en distingue par une feuille plus grande, membraneuse et cunéiforme courtement acuminée (0.3-0.4 mm). Le port de T. cauliflora (petit arbre de 4-7 m de hauteur) est proche de T. sericea mais la nouvelle espèce s'en distingue par des fleurs petites $(1-1,6 \mathrm{~cm} \times 1,5-2,2 \mathrm{~mm})$, glabres, membraneuses et des fruits pubescents, petits $(0,9-1,5$ $\times 0,8 \mathrm{~cm}$ ), globuleux possèdant 10 à 12 valves.

Paratypi. - Madagascar. Prov. Toliara : forêt d'Analavelona, [22 42'22”S 4409'10”E], 1033 m, 5.XII.2015, fr., Andriamihajarivo, Rakotoarivelo छ Bruno 1962 (MO, P, TAN); ibid. loc., [22³9'12”S 44¹1'40”E], 1057 m, 3.XII.2016, fl., Andriamihajarivo \& Rakotoarivony 2044 (MO, P,

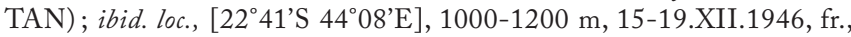
Humbert 19720 (P,TEF); ibid. loc., [2241'22"S 4409'39”E], 967 m, 1.II.2012, fr., Randrianarivony et al. 463 (G, MO, TAN); ibid. loc., [22 41'13”S 4409'28”E], 987 m, 3.XII.2015, fr., Randrianarivony et al. 750 (MO, P,TAN).

\section{Remerciements}

Nous remercions le Gouvernement de Madagascar par le biais du Ministère de l'Environnement, de l'Ecologie et de la Forêt (MEEF) pour l'octroi des permis de recherche, Faranirina Lantoarisoa pour la préparation des ces permis, Roger Lala Andriamiarisoa pour la magnifique illustration, et Fano Rajaonary pour la réalisation de la carte de distribution. Nous sommes très reconnaissants envers toutes les équipes travaillant pour la conservation de la forêt d'Analavelona pour les efforts fournis sur le terrain afin de récolter l'espèce nouvelle décrite ici. Nous remercions finalement Sven Buerki pour ses commentaires avisés qui ont amélioré une version antérieure de ce manuscrit. 


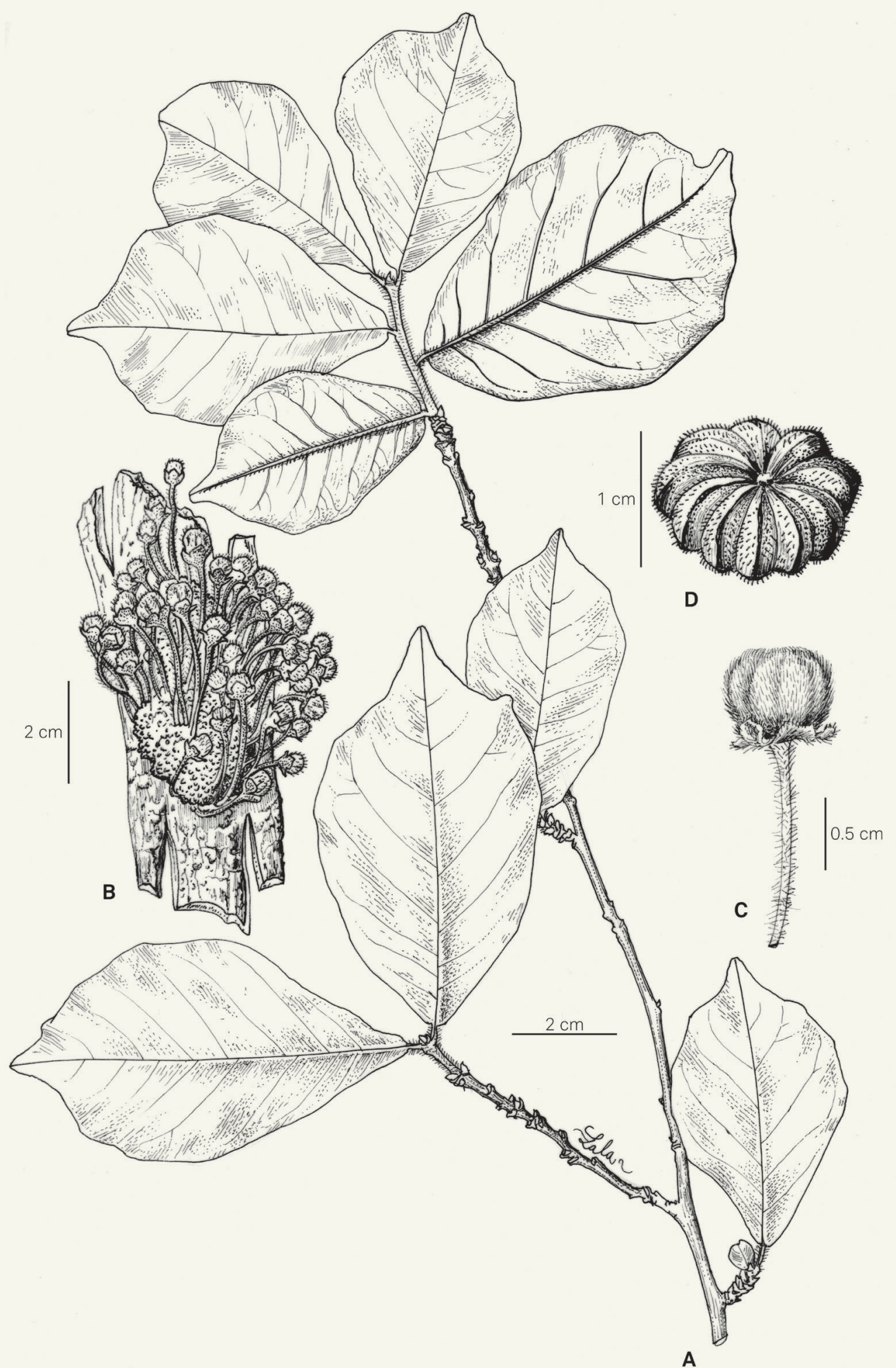

Fig. 2. - Turraea cauliflora J.-F. Leroy \& Lescot ex Randrianari. \& Callm. A. Rameau; B. Fruits immatures; C. Détail d'un fruit immature; D. Fruit mature.

[A, D: Randrianarivony et al. 463, TAN; B, C: Service Forestier 22188, TAN] [Dessin: R.L. Andriamiarisoa] 

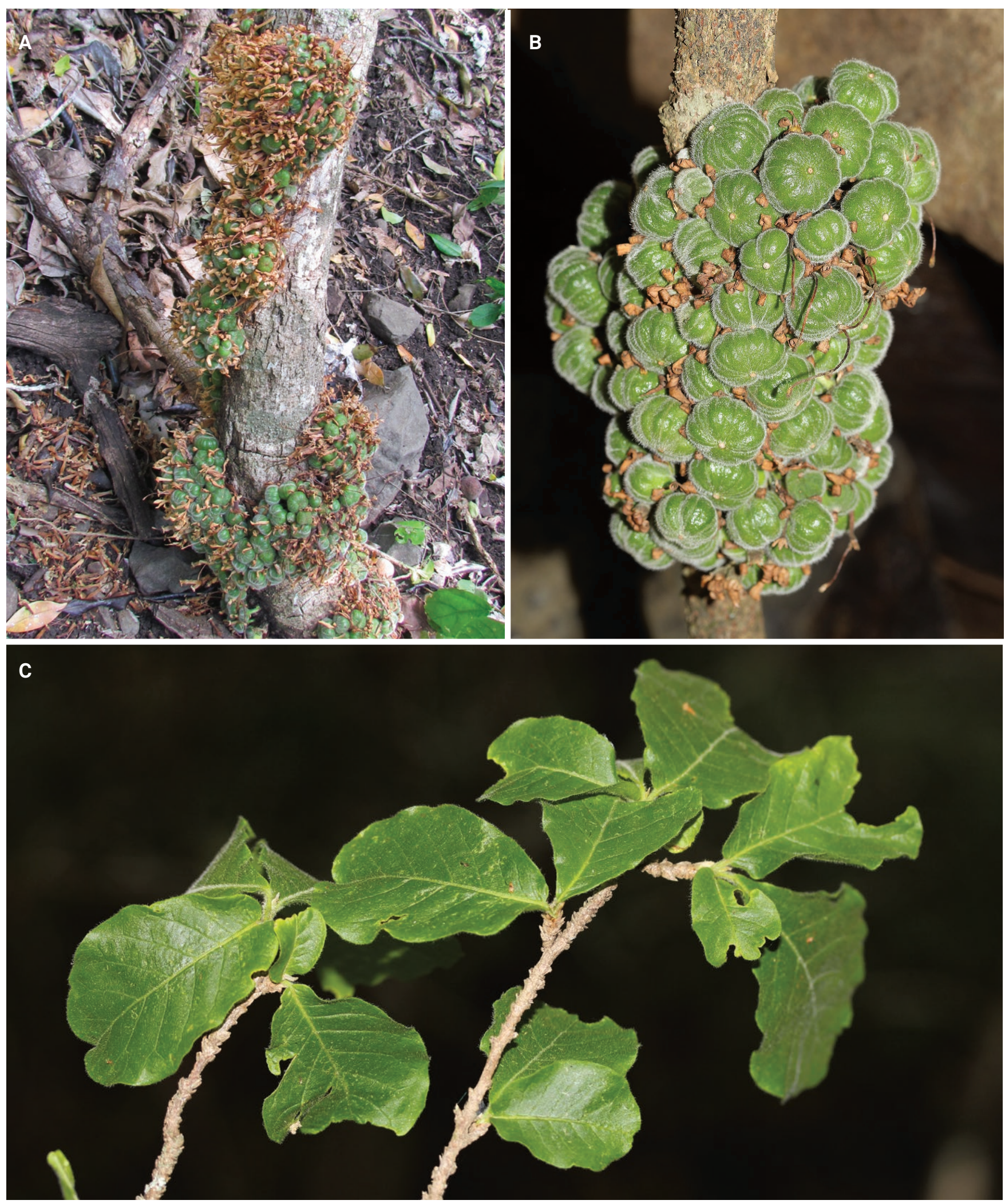

Fig. 3. - Turraea cauliflora J.-F. Leroy \& Lescot ex Randrianari. \& Callm.

A. Fructification à la base du tronc; B. Détail des fruits. C. Rameaux.

[Photos: A: A. Andriamihajarivo; B-C: F. Rakotoarivony] 


\section{Références}

Andriamihajarivo, T.H. (2014). Document de création définitive, nouvelle aire protégée: forêt sacrée Analavelona. Missouri Botanical Garden, Antananarivo.

Andriamihajarivo, T.H., A. Ravoahangy, T. Rakotoarison \& M. Andrianarinala (2008). Evaluation biologique et sociologique en vue de création en Nouvelle aire protégée de la forêt sacrée d'Analavelona - Sakaraha. Missouri Botanical Garden, Antananarivo.

APC (2017). The Australian Plant Census. Australian National Botanic Gardens, Australian Government, Canberra [http://www.anbg. gov.au/chah/apc/index.html].

APD (2017). The African Plant Database. Conservatoire et Jardin botaniques de la Ville de Genève and South African National Biodiversity Institute, Pretoria [http ://www.ville-ge.ch/musinfo/ $\mathrm{bd} / \mathrm{cjb} /$ africa]

Bosser, J. (2002). Une nouvelle espèce de Turraea (Meliaceae) des Mascareignes. Localisation de T. thouarsiana et identité de T. casimiriana. Adansonia ser. 3, 24:113-116.

Callmander, M.W., P.B. Phillipson \& P.P. Lowry II (2012). Novelties from the Northern Mountains Complex of Madagascar. III. Two new species of Turraea L. (Meliaceae). Adansonia ser. 3, 34: 93-102.

Horning, N.R (2004). The Limits of rules: When rules promote forest conservation and when they do not - Insights from Bara Country, Madagascar. PhD thesis, Department of Government Cornell University, NY.

IUCN (2012). IUCN Red List Categories and Criteria : version $3.1 \mathrm{ed}$. 2. IUCN Species Survival Commission, Gland and Cambridge.
Mabberley, D.J., C.M. Pannell \& A.M. Sing (1995). Meliaceae. Fl. Males. 12(1).

Madagascar Catalogue (2017). Catalogue of the plants of Madagascar. Missouri Botanical Garden, St. Louis and Antananarivo [http://www.efloras.org/madagascar].

MEEMF [Ministère dE L'Environnement, DE L'Ecologie, dE LA Mer et des Forêts] (2015). Décret N $N^{\circ} 2015-766$ portant création de l'Aire Protégée dénomée "Forêt sacrée Alandraza Analavelo" communes rurales Amboronabo, Mababoboka et Mikoboka, District de Sakaraha, Région Atsimo-Andrefana. Antanananarivo.

Moat, J. \& P. Smith (2007). Atlas of the vegetation of Madagascar. Kew Publishing.

Pennington, T.D. \& B.T. Styles (1975). A generic monograph of the Meliaceae. Blumea 22: 419-550.

RANDRIANARIVONy, T.N. (2015). Etudes éthnobotaniques et écologiques des plantes utiles de la nouvelle Aire Protégée d'Analavelona (District de Sakaraha). Thèse de doctorat, Université d'Antananarivo, Madagascar.

Randrianarivony, T.N., T.H. Andriamihajarivo, A.V. Ramarosandratana, F. Rakotoarivony, V.H. Jeannoda, A. KuHLman, A. Randrianasolo \& R. Bussmann (2016). Value of useful goods and ecosystem services from Agnalavelo sacred forest and their relationships with forest conservation. Madagascar Conservation Developm.11: 44-51.

Scott, A.J. \& J. Bosser (1997). Meliaceae. Fl. Mascareignes 69.

Sourdat, M. (1976). Le Sud-ouest de Madagascar, étude géodynamique. Cahier ORSTOM, Série Pédologique 14: 245-251. 